\title{
DRIVER PERFORMANCE WHILE INTERACTING WITH THE 511 TRAVEL INFORMATION SYSTEM IN URBAN AND RURAL TRAFFIC
}

\author{
Laura M. Stanley, Michael J. Kelly, Suzanne Lassacher \\ Western Transportation Institute \\ Montana State University \\ Bozeman, Montana, USA \\ E-mail: mkelly@coe.montana.edu
}

\begin{abstract}
Summary: The national "511" highway information system is heavily used by drivers, especially during inclement weather, to plan and replan their trips. Few studies have explored the safety and usability of the 511 user interface, especially in the context of a mobile phone user who has the added workload of driving a vehicle. In this study, 36 drivers were divided into three groups (hand-held cell phone, hands-free cell phone, and control group) and drove a series of urban and rural scenarios in a high fidelity driving simulator. Drivers in the cell phone groups interacted with the Montana 511 travel information system to obtain road information on a segment of highway. Performance on the primary driving task (e.g., lanekeeping and speed control) was not affected by use of the 511 traveler information system. Driving tasks that required urgent attention (e.g., responding to unexpected traffic conflicts) were degraded by using the 511 travel system regardless of the type of phone used. Drivers using either cell phone to interact with the 511 information system were found to have a higher number of collisions and less situation awareness than those not interacting with the 511 system. Drivers using a hand-held cell phone were also found to have a higher frequency of braking responses. The increased crash risk of the phone users in our study (3.0 - 3.8) was very comparable to that reported by earlier studies of the risk of cell phone conversations.
\end{abstract}

\section{INTRODUCTION}

The burgeoning availability of information and communication devices for use in vehicles has become a double-edged sword, with both significant benefits and significant costs. Mobile telephones allow motorists to maintain social contacts, conduct and coordinate business, make restaurant and theatre reservations, and even track their stock portfolios. Traveler information such as lodging availability and driving directions can be sought from within the vehicle.

Recently most states have been implementing a "Dial 511" travel information system. This system will allow motorists to obtain advanced information on road conditions, adverse weather, traffic incidents, and construction along their planned route by dialing 511 on their telephones and interacting with an automated system. Providers of the 511 systems have emphasized that drivers should call to obtain this information before departure or park their cars safely along the route to make the call. Many drivers, however, access the system to update their travel information while they are driving. No studies to date have examined the safety of using mobile telephones to acquire such travel information. 
Numerous recent studies have examined the potential effects on driver performance and resulting risks related to cell phone conversations while driving. Anecdotal reports of vehicle accidents caused by drivers who were dialing, answering, or conducting mobile telephone calls suggest that the distractions and added workload of telephone use may add a significant increment of risk. In a recent survey by Thulin and Gustafsson (2004) drivers admitted to missing exits, failing to see traffic signals, losing control of their speed, and experiencing near-crashes while using their mobile phones. None of the drivers reported an actual accident, but it was estimated that 10-20 Swedish fatalities per year result from the use of mobile phones while driving.

In a widely cited earlier study, Redelmeier and Tibshirani (1997) examined the telephone billing records of approximately 700 drivers who had experienced property damage crashes. They found that drivers who were using their phones within 10 minutes before an accident had a risk factor approximately 4 times that of non-phone users. The investigators subsequently reported that, due to a number of limitations on their original experimental design, these numbers are probably underestimates of the actual risk (Redelmeier and Tibshirani, 2001). Green (2000) summarized studies of crash frequency and cell phone use and concluded that making a single call during a trip approximately triples the crash risk.

Primary driving tasks such as simple lane and speed maintenance typically are not significantly impacted by a communication task (Horrey and Wickens, 2004a). A recent study by Strayer, et al. (2005), however, found that drivers engaged in cell phone conversations had significantly slower reaction times to urgent events than drivers not engaged in cell phone conversations. It was hypothesized that drivers engaged in cell phone conversations are less aware of the environment around them. Horrey and Wickens (2004b) postulated that the primary driving tasks involve different attentional resources (ambient visual channels) than do responses to unexpected events (focal visual channels). Communication tasks may interfere more with processing in the focal channels and differentially degrade performance on tasks utilizing those channels.

It should be noted that the task of acquiring information using the 511 traveler information system via a cellular phone differs from that of conversing and interacting on a cellular phone. The 511 task is not a free conversation. The user must acquire information via a menu-based system. The purpose of this research was to determine whether using the 511 system has the same impacts on driver performance and safety as a free conversation via the cell phone.

\section{RESEARCH METHODS}

\section{Subjects}

The subjects were 36 licensed drivers between the ages of 18 and 63 years (mean age $=31$ ) who were recruited by announcements on the university campus and in the surrounding community. All subjects were cellular telephone users. Subjects were compensated for their participation in the research and received a bonus for completing testing without experiencing a crash. Potential subjects completed a screening questionnaire to identify and disqualify those who had medical conditions or histories that might indicate increased levels of risk (e.g., headaches and motion sickness) in the simulation environment. All 36 subjects who began testing completed the study although four reported some symptoms of motion discomfort. 


\section{Laboratory Equipment}

Simulator. Data were collected using the Western Transportation Institute's Driving Simulation Laboratory. This laboratory is a 36-square-meter light and sound-controlled room containing a DriveSafety 500C simulator running HyperDrive ${ }^{\mathrm{TM}}$ Simulation Authoring Suite software and Vection $^{\text {TM }}$ simulation software version 1.9.7. The simulator is comprised of a partial 1996 Saturn SL sedan cab with fully functional controls, five rear projection plasma displays arranged in a semicircle around the front of the cab providing a 150-degree field of view and on-screen rearview mirrors, four audio speakers, vibration generator, a simulator programmer/operator station, and seven associated computers to generate the scenarios, visual and auditory environment, and collect data.

The simulator provides physics-based vehicle dynamics. The graphics systems render realistic driving scenarios, including geometrically correct urban and rural roadways, traffic control devices, cultural features, ambient traffic, pedestrians, animals and other features. Realistic auditory effects of traffic, engine noise, and wind noise are generated by the 3-D audio system.

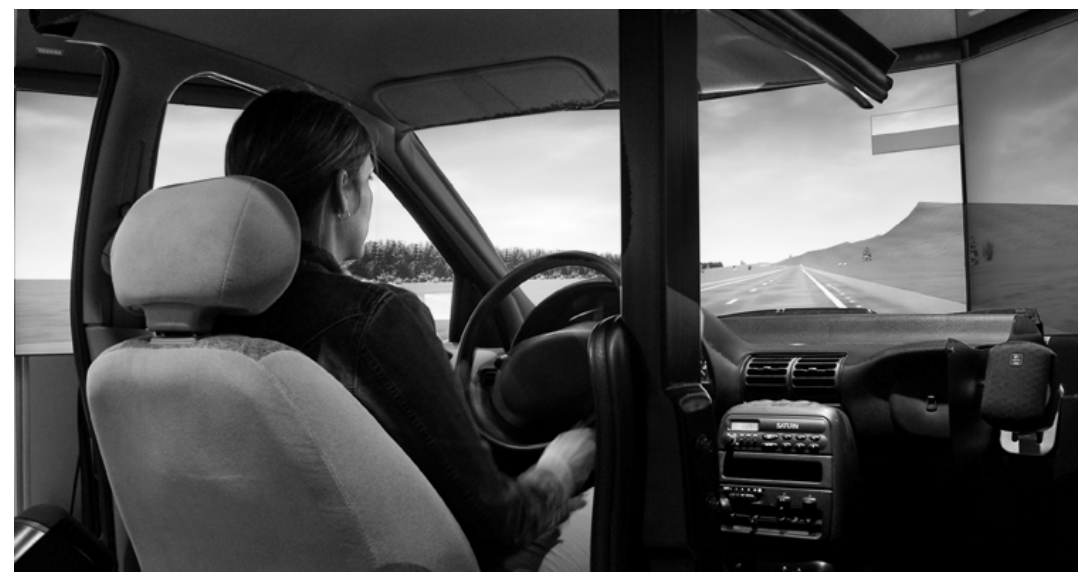

Figure 1. DriveSafety DS500C Vection Driving Simulator

Telephone. The telephone was a standard Motorola V120C hand-held cell phone. A Plantronics headphone with a single ear cup and boom microphone was used for hands-free operation.

\section{Procedures}

Prior to testing sessions, subjects completed screening questionnaires directed primarily at identifying susceptibility to simulator-induced discomfort (SID), including nausea, headaches, and dizziness. Subjects were then acclimated to the driving simulator by completing a series of six, three to five-minute training scenarios in the simulator. Training began with relatively gentle drives designed to minimize SID. As subjects proceeded through the training, the scenarios became longer, more challenging, and more visually complex. Subjects were then trained and given practice using the Montana Department of Transportation's 511 highway information line, including the voice understanding system. At the completion of training, subjects completed a follow-up questionnaire on any SID symptoms they might have experienced. 
The testing session was conducted one to two days after the training session. For testing, subjects were divided into three groups of 12 each: Hand Held Phone, Hands Free Phone, and Control. The groups were equalized in terms of gender and mean age. Subjects drove a series of four, 6.5minute scenarios. Two of the scenarios had cultural features, traffic control devices, and ambient traffic typical of an urban environment, while the other two had features typical of a rural driving environment. Common traffic conflicts were programmed into the testing scenarios including vehicles entering traffic unexpectedly, pedestrians crossing the street, and large animals entering the roadway. The order of scenario presentation was randomized among subjects within the groups.

Subjects were given instructions to obey all traffic signs, maintain a safe following distance (approximately 2 seconds), and drive as they normally would. Those in the cell phone groups were given a task to gather road and weather conditions for a particular segment of Interstate 90 using Montana's 511- telephone traveler information system employing voice commands to navigate the system menus. The experimenter prompted subjects when to begin dialing. Subjects disconnected the phone at the completion of the task.

After each scenario, subjects were given a situation awareness questionnaire in which they were asked a series of ten questions regarding objects they remembered seeing while driving (e.g., an ambulance). At the end of the test session, subjects completed a questionnaire related to their experience with simulator discomfort (if any) and a usability survey on the 511-traveler information system.

\section{RESULTS AND DISCUSSION}

All subjects made all turns as instructed and properly stopped at all signaled intersections (i.e., no red-light running). Several errors were made in using the 511-traveler information systems, primarily due to the system's voice-understanding limitations. The number of errors was not recorded due to the lack of an interface that would enable the experimenter to hear the interactions between the subject and the 511-system. To address this inadequacy, at the end of the session subjects were asked to report and describe any errors made. Ninety-four percent reported making errors due to the voice-understanding software.

The dependent variables relating to driver performance were analyzed. These include velocity, root-mean-square values of lane position, steering, acceleration, braking, lateral and longitudinal acceleration, number of collisions, and maintenance of speed limit. Collisions was a count of how many objects the subject collided with while driving. Maintenance of the speed limit included the subject's velocity minus the posted speed limit at that frame. The root-mean-square (RMS) value for lane position, steering, acceleration, braking, lateral and longitudinal acceleration were used in the analysis.

Data analyzed for the cell phone groups included that collected during the phone task; data analyzed for the control group included that collected between average start (43 seconds into the scenario) and end time of the cell phone ( 5 min into the scenario). The average time on the phone was approximately 4.5 minutes. The number of incorrect responses on the situation awareness probe was used to assess the subjects' awareness of the driving environment. 
Two types of analyses were conducted: driver performance measurement and situation awareness. For the driving performance data, excluding the number of collisions, mean differences on each dependent variable as a function of the experimental condition was conducted using a 3 (Task Condition: Control, Hands-free Cell Phone, Hand-held Cell Phone) x 4 (Environment: Rural 1, Rural 2, Urban 1, Urban 2) analysis of variance (ANOVA). In assessing situation awareness and the number of collisions, the Kruskal-Wallis one-way analysis of variance by ranks was performed on the number of incorrect responses and the number of collisions. In each environment, subjects completed two tests in that environment (i.e. two rural and two urban scenarios). The ANOVA was completed separately for each driving environment; no aggregation was conducted because the scenarios were different from each other.

\section{Driving Performance}

For the braking RMS, a measure of braking activity, significant main effects $(\mathrm{p}<.05)$ were found for task condition. The mean braking RMS for the hand-held cell phone condition $(\mathrm{M}=$ $0.1196)$ was significantly higher $(\mathrm{p}<.05)$ than the for the control condition $(\mathrm{M}=0.0991)$. Results are shown in Figure 2.

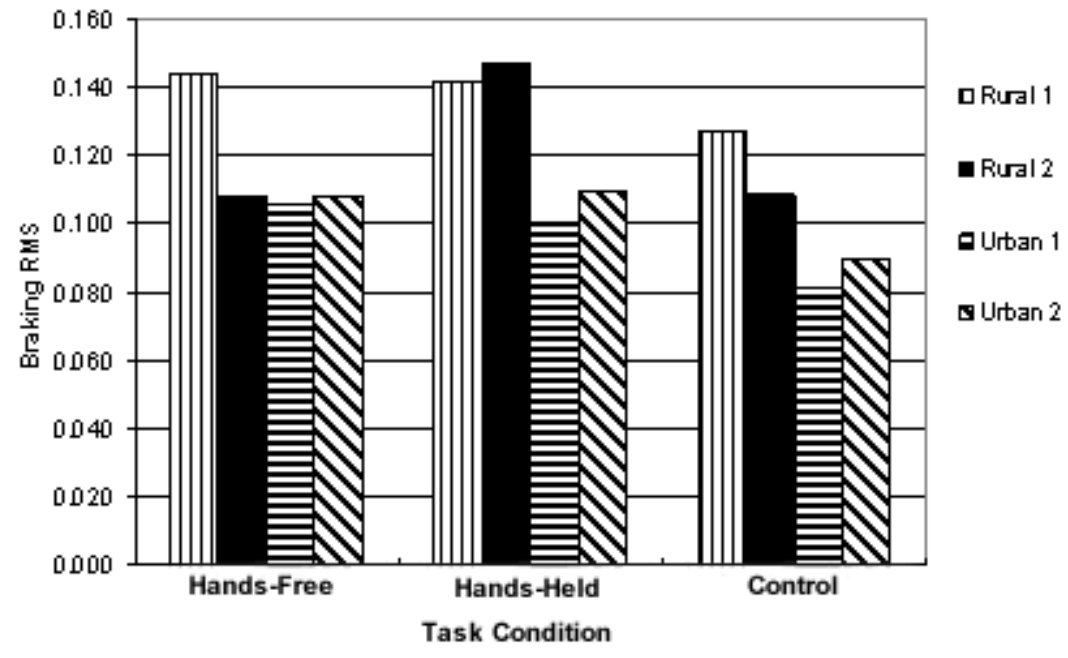

Figure 2. Braking RMS across groups and driving environments

For the number of collisions, significant differences $(\mathrm{p}<.05)$ were found among task conditions. The number of collisions for the hand-held cell phone condition $(\mathrm{M}=4.75)$ and the hands-free condition $(M=3.75)$ was significantly higher $(\mathrm{p}<.05)$ than for the control condition $(\mathrm{M}=1.25)$, as shown in Figure 3.

\section{Situation Awareness}

The number of incorrect responses had marginally significant effects among task condition, $\mathrm{X}^{2}=$ $2.15, \mathrm{p}<.10$. The mean number of incorrect responses for the hand-held condition $(\mathrm{M}=16.5)$ and the hands-free condition $(\mathrm{M}=16.25)$ was marginally higher $(\mathrm{p}<.10)$ than that for the control condition $(\mathrm{M}=13.75)$, as shown in Figure 4. 


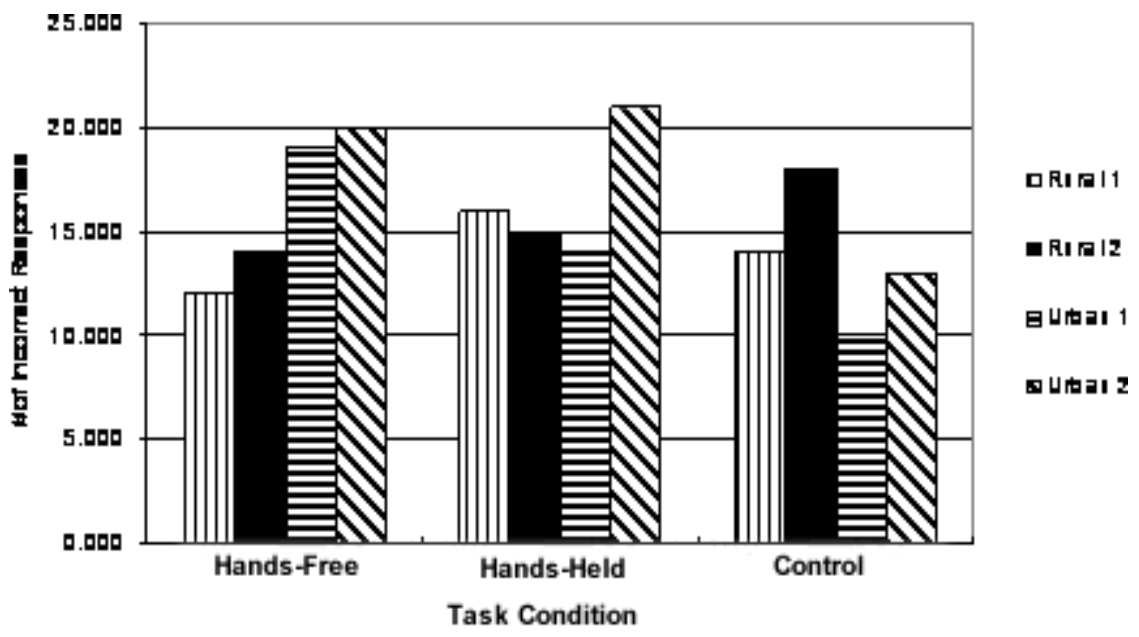

Figure 3. Number of collisions across groups and driving environments

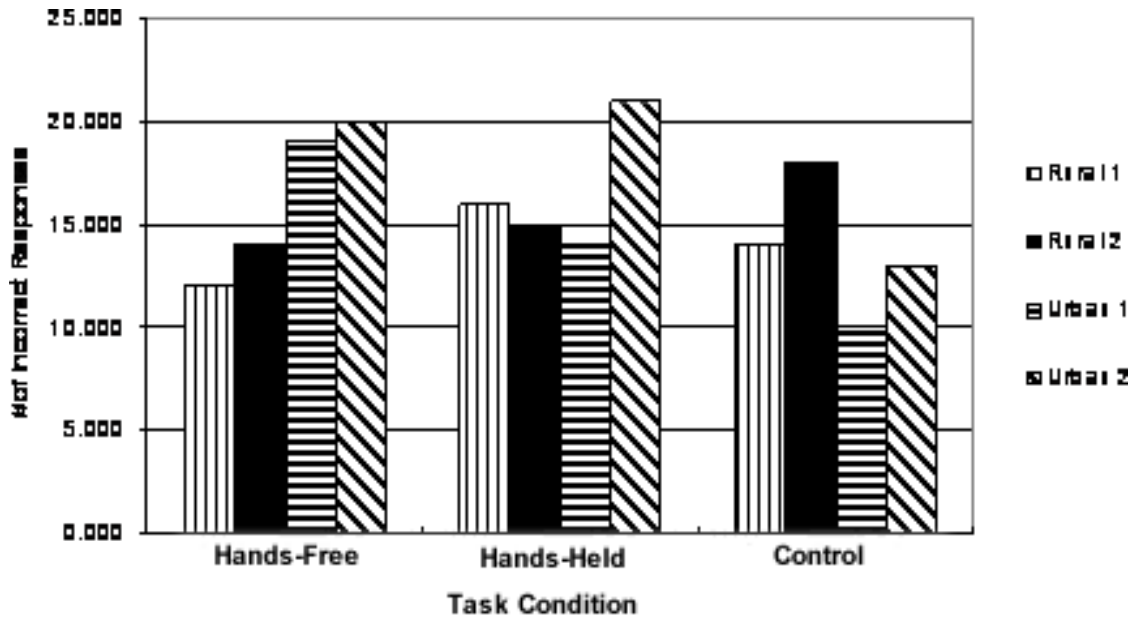

Figure 4. Number of incorrect responses across groups and driving environments

\section{CONCLUSIONS}

Acquiring information using the 511 traveler information system via a cellular phone is different than conversing and interacting with a cellular phone. Subjects did not engage in a conversation at any point during their task. Rather they used voice commands to determine what the road and weather conditions were for the given segment of highway.

Despite these differences, results from this study were strikingly similar to findings from studies of cell phone conversations while driving. Interaction with the 511 travel information system appears to have the same performance effects and risks as a free cell phone conversation. Research using a broad range of methodologies including accident epidemiology, field studies, and simulation studies has indicated that cell phone use increases accident risk by a factor of 3 to 4. Our results using the 511 interaction task (risk factor of 3.0 to 3.8) duplicated those results. 
Furthermore, few studies have found the anticipated safety benefit of a hands-free telephone interface over a hand-held interface. This study agreed. We found, at most, a marginal safety benefit for the hands-free interface.

Performance on the primary tasks of driving (e.g., lane and speed maintenance) was found to be unaffected by interacting with the cell phone. Yet the tasks that require more prompt response times (e.g., avoiding collisions during unexpected conflicts) were degraded by the use of a cell phone, regardless of the type of instrument used. This finding is typical of driving simulation and test track research (Horrey and Wickens, 2004a).

It appeared that drivers were less aware of their surroundings when interacting with the 511 traveler information system while using a cellular phone and driving. Our drivers who communicated with 511 performed more poorly in recalling target objects in the environment than did their counterparts without communication tasks. This finding is consistent with other studies reporting a decrement in visual attention and shrinkage of the field of view by phone users (e.g., Atchley and Dressel, 2004).

\section{REFERENCES}

Atchley, P. \& Dressel, J. (2004). Conversation limits the functional field of view. Human Factors, 46(4): 664-673.

Green, P. (2000). Crashes induced by driver information systems and what can be done to reduce them (SAE Paper 2000-01-C008). Warrendale, PA: Society of Automotive Engineers.

Horrey, W.J. and Wickens, D.D. (2004a). The impact of cell phone conversations on driving: $A$ meta-analytic approach (Technical Report AHFD-04-2/GM-04-1). Savoy, IL: University of Illinois at Urbana-Champaign.

Horrey, W.J. and Wickens, C.D. (2004b). Driving and side task performance: The effects of display clutter, separation, and modality. Human Factors, 46(4): 611-624.

Redelmeier, D.A. and Tibshirani, R.J. (1997). Association between cellular telephone calls and motor vehicle collisions. New England Journal of Medicine, 336(7), 453-458.

Redelmeier, D.A. and Tibshirani, R.J. (2001). Car phones and car crashes: Some popular misconceptions. Canadian Medical Association Journal, 164(11): 1581-1582.

Strayer, D. L. \& Drews, F. A. Crouch, D. J., \& Johnston, W. A. (2005). Why do cell phone conversations interfere with driving? In: W. R. Walker and D. Herrmann (Eds.) Cognitive Technology: Essays on the Transformation of Thought and Society. Jefferson, NC: McFarland \& Company, Inc.: 51-68.

Thulin, H. and Gustafsson, S. (2004). Mobile phone use while driving: Conclusions from four investigations (VTI rapport 490A). Linkoping, Sweden: Swedish National Road and Transport Institute. 